\title{
Estudo de viabilidade sobre o uso de Chatterbots em redes sociais para acessar ambientes de e-learning
}

\author{
Flávia Linhalis Arantes ${ }^{1}$, Fernanda Maria Pereira Freire ${ }^{1}$, Bruno Simioni ${ }^{2}$ \\ ${ }^{1}$ Núcleo de Informática Aplicada à Educação (NIED) - Universidade Estadual de \\ Campinas (UNICAMP) - Campinas - SP - Brasil \\ ${ }^{2}$ Empresa Ampliato - Campinas - SP - Brasil \\ farantes@unicamp.br, ffreire@unicamp.br, bruno@ampliato.com.br
}

\begin{abstract}
The use of social networks in conjunction with e-learning platforms has been explored in order to create more interesting and collaborative learning environments for students. This synergy has become a new trend in the deployment of Digital Information and Communication Technologies in education. However, the existing literature explores how learning environments can be enriched by social networks or social tools. In our work, we propose to adopt the opposite - we use social networks to access or explore an e-learning environment. In this article we present a feasibility assessment on the use of the WAS (Write and Solve) agent to access features of the TelEduc e-learning environment. To perform the evaluation we applied a questionnaire to TelEduc users. The results show that the integration between the agent and the e-learning environment is desired and considered useful by the respondents.
\end{abstract}

Resumo. O uso conjunto de redes sociais e plataformas de e-learning tem sido explorado com o intuito de criar ambientes de aprendizagem mais interessantes e colaborativos para os alunos, constituindo uma nova tendência de uso das Tecnologias Digitais de Informação e Comunicação (TDIC) na educação. No entanto, os trabalhos encontrados na literatura partem de ambientes de aprendizagem que são enriquecidos pelas redes sociais ou por ferramentas sociais. Em nosso trabalho, propomos adotar o caminho inverso isto é, usar as redes sociais para acessar elou explorar um ambiente de elearning. Neste artigo apresentamos uma avaliação de viabilidade sobre o uso do agente WAS (Write and Solve) para acessar funcionalidades do ambiente de e-learning TelEduc. Para realizar a avaliação aplicamos um questionário aos usuários do TelEduc. Os resultados mostram que a integração entre o agente e o ambiente de e-learning é desejada e considerada útil pelos respondentes.

\section{Introdução}

A web pode ser considerada um fenômeno social que diminui distâncias e agrupa pessoas em diferentes tipos de redes sociais, com diferentes propósitos. Trata-se de um movimento crescente, desencadeado pelo barateamento de dispositivos - computadores pessoais, tablets, smartphones - e pela melhoria das condições de acesso à Internet alcançada por muitos países, fomentando a inclusão digital de um maior número de 
pessoas. As redes sociais têm desempenhado um papel fundamental na maneira como as pessoas utilizam a Internet nos dias de hoje. Atualmente, as pessoas, em geral, usam as redes sociais como espaço de interação, de encontro e de entretenimento.

Alguns trabalhos na literatura tematizam sobre o uso das redes sociais em conjunto com as plataformas de e-learning com o objetivo de criar ambientes mais interessantes para a aprendizagem dos alunos. As características intrínsecas das redes sociais, tais como colaboração, compartilhamento e comentários contribuem para a criação de ambientes ativos e motivadores para os estudantes [Li \& Liu 2009; Figl et. al. 2008], que são encorajados a compartilhar e a interagir. De acordo com Rodrigues e colegas (2010), levar serviços semelhantes aos disponíveis na web 2.0 para o ambiente educacional, através de uma plataforma de e-learning mais ativa e colaborativa, pode melhorar a aprendizagem dos alunos.

Realizamos um estudo bibliográfico com o propósito de identificar como a integração entre os ambientes de e-learning e as redes sociais está sendo posta em prática. Com base nesse estudo, observamos que as soluções criadas para agregar recursos comumente presentes nas redes sociais aos ambientes de e-learning, podem ser divididas em duas categorias:

a) Criação de ambientes de aprendizagem que incorporam recursos das redes sociais ou que têm estrutura e ferramentas que permitem mais interação entre seus participantes. Esse é o caso do TelEduc ${ }^{1}$ [Cerceau 1998], Redu ${ }^{2}$ e $\mathrm{NAVi}^{3}$. Outros trabalhos nessa mesma linha são descritos em Halimi et. al. (2011), Kaeomanee et. al. (2012), Colazzo et. al. (2011) e Rodrigues et. al. (2010).

b) Adaptação de ambientes de aprendizagem já existentes de modo a incorporar ferramentas mais interativas. Como exemplos podemos citar MOT 2.0 [Ghali \& Cristea 2009], Blackboard ${ }^{4}$, Angel $^{5}$ e Sakai ${ }^{6}$.

É cada vez mais comum a reivindicação do uso das redes sociais pelos alunos que utilizam ambientes de e-learning. O público que temos hoje nas escolas e no mundo usa as redes sociais como espaço de interação, encontro, entretenimento e, até mesmo, para mobilização sócio-política. Apesar disso, tanto a solução a) quanto a b) apresentadas acima partem de ambientes de aprendizagem que são enriquecidos com recursos encontrados nas redes sociais e/ou por ferramentas sociais. Nenhuma delas adota o caminho contrário - isto é, usar as redes sociais para acessar e/ou explorar um ambiente de e-learning. Supomos que soluções que exploram esse caminho podem ter um impacto bastante positivo e motivador para um público que "vive", cada vez mais, nas redes sociais.

Um exemplo de aplicação que usa as redes sociais para acessar outros ambientes é o WAS (Write and Solve) ${ }^{7}$. O WAS é um agente de software que utiliza

\footnotetext{
${ }^{1} \mathrm{http}: / /$ www.teleduc.org.br/

${ }^{2}$ http://redu.com.br/

${ }^{3} \mathrm{http}: / /$ www.softwarepublico.gov.br/ver-comunidade?community id=60993607. Acesso em 30/07/2013.

4 www.blackboard.com. Acesso em 30/07/2013.

${ }^{5} \mathrm{http}$ ://www.angellearning.com. Acesso em 30/07/2013.

6 www.sakaiproject.org. Acesso em 30/07/2013.

7 http://www.ampliato.com.br. Acesso em 30/07/2013.
} 
comunicadores instantâneos para conectar usuários a outros sistemas e, assim, coletar e inserir informações. $\mathrm{O}$ agente simula um ser humano que interage por meio de perguntas e respostas em linguagem natural. $\mathrm{O}$ agente pode ser adicionado à lista de contatos dos usuários nos comunicadores das principais redes sociais (Facebook Chat, Twitter, Gtalk, etc.) assumindo, assim, a aparência de um "amigo" a quem o usuário pode fazer perguntas e obter respostas como se estivesse conversando com uma pessoa. $\mathrm{O}$ agente apresenta uma versão pública integrada a serviços como Buscapé ${ }^{8}$, LinkedIn ${ }^{9}$ e FourSquare $^{10}$. A Figura 1 mostra um exemplo de interação com o WAS.

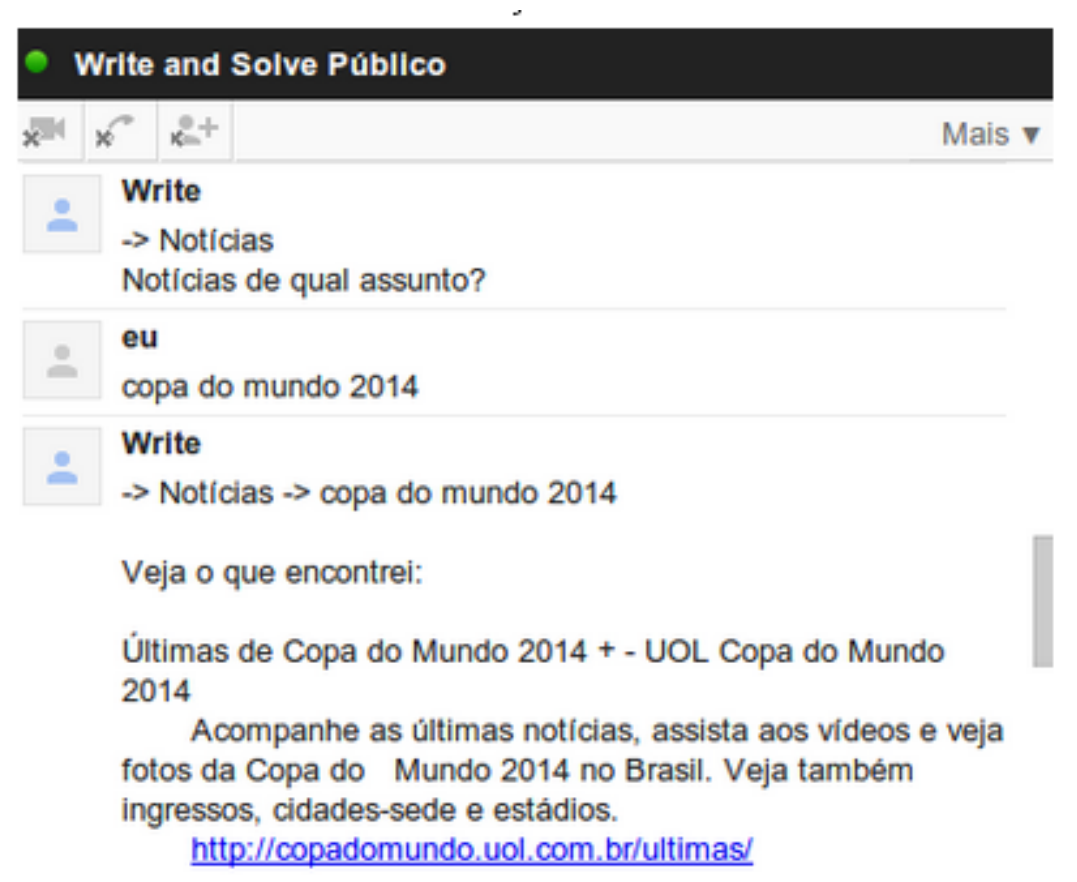

\section{Figura 1. Exemplo de interação entre um usuário e o Google Custom Search, intermediada pelo WAS.}

O WAS é um exemplo de agente do tipo Chatterbot - isto é, um programa de computador criado para simular uma conversa humana. Esse tipo de agente processa uma entrada em linguagem natural e consulta uma base de conhecimento para dar respostas que imitam as respostas humanas [Mahapatra et. al. 2012].

Neste artigo investigamos a utilização de agentes do tipo Chatterbots em contextos educacionais e apresentamos uma avaliação de viabilidade sobre o uso do agente WAS para acessar funcionalidades do ambiente de e-learning TelEduc. Tal avaliação foi elaborada com o objetivo de conhecer a opinião dos usuários sobre a necessidade e a utilidade desse tipo de recurso em ambientes de e-learning, fator esse não relatado na literatura consultada (cf. seção 2).

Este artigo está organizado da seguinte maneira: na Seção 2 descrevemos alguns trabalhos encontrados na literatura que exploram a utilização de Chatterbots em contextos educacionais. Na Seção 3 apresentamos a avaliação de viabilidade da

\footnotetext{
${ }^{8}$ www.buscape.com.br. Acesso em 30/07/2013.

${ }^{9}$ br.linkedin.com. Acesso em 30/07/2013.

${ }^{10} \mathrm{https}$ ://foursquare.com. Acesso em 30/07/2013
} 
integração do agente WAS com o ambiente de e-learning TelEduc que, supomos, pode ser generalizada para outros ambientes de e-learning. Na Seção 4 apresentamos uma discussão sobre a avaliação de viabilidade realizada, destacando alguns pontos que nortearão a integração do agente ao ambiente de e-learning. Finalmente, a Seção 5, apresenta as conclusões do artigo e os direcionamentos para trabalhos futuros.

\section{Chatterbots em Contextos Educacionais}

Chatterbots têm sido utilizados para solucionar diversos tipos de problemas, representando um paradigma alternativo aos softwares tradicionais. O primeiro Chatterbot, ELIZA ${ }^{11}$, foi criado em 1966 [Weizenbaum 1966]. Sua ideia principal era reconhecer palavras e frases relevantes na entrada do usuário e gerar respostas significativas para dar continuidade à interação. Seguindo uma abordagem semelhante, outros Chatterbots foram desenvolvidos e refinados, sendo o A.L.I.C.E. (Artificial Internet Linguística Computer Entity) o mais bem sucedido [Wallace 2009]. Atualmente, há uma variedade de Chatterbots desenvolvidos para os mais diversos domínios, tais como: jogos interativos [Sing et. al. 2006], serviços de informação [Weerawarna et. al. 2011; Cassell 2001], e-government [Mahapatra et. al. 2012], ecommerce [Takegata \& Tanaka-Ishii 2010], suporte médico [Bickmore \& Giorgino 2006], educação [Oliveira et. al. 2012; Moreno et. al. 2012; Batista et. al. 2009; Mikic et. al. 2009], dentre outros. Nesta seção, descrevemos alguns Chatterbots utilizados em ambientes de e-learning.

O trabalho de Batista e colegas apresenta um Chatterbot que pode ser usado em Ambientes Virtuais de Aprendizagem (AVAs) para guiar o aluno na obtenção de explicações relacionadas ao FAQ [Batista et. al. 2009].

O AVA INES oferece o CHARLIE, um Chatterbot inteligente, capaz de se comunicar com os alunos em linguagem natural para ajudá-los em suas tarefas e para oferecer conteúdo específico [Mikic et. al. 2009].

O trabalho de Moreno e colegas (2012) apresenta uma aplicação desenvolvida para ambientes móveis que utiliza um Chatterbot para encontrar e acessar objetos de aprendizagem. O Chatterbot encontra padrões nos diálogos e os utiliza para encontrar os objetos de aprendizagem.

O trabalho de Oliveira e colegas apresenta o I-Collaboration 3.0 [Oliveira et. al. 2012], que cria espaços virtuais de aprendizagem, conectando os usuários em diferentes ferramentas da Web 2.0. O I-Collaboration 3.0 possibilita um cenário onde o Twitter, o MSN, o Facebook e o AVA Moodle são integrados. Isso é, uma instância de um agente inteligente estará disponível em cada um desses ambientes (como um contato no MSN, como um usuário no Twitter e como um Chatterbot no AVA). Dessa forma, supondo que o aluno interaja com o agente através do Facebook sobre conceitos relacionados à programação e, em seguida, interaja com o agente através do MSN, o agente saberá que o aluno já se comunicou com ele através do Facebook e demonstrou interesse em conceitos relacionados à programação.

\footnotetext{
${ }^{11} \mathrm{http}: / /$ nlp-addiction.com/eliza/
} 
Uma característica importante do WAS é que ele está presente nos comunicadores instantâneos das redes sociais, o que o torna facilmente acessível aos usuários. Dentre os trabalhos pesquisados, o único que também apresenta essa característica é o I-Collaboration 3.0 [Oliveira et. al. 2012]. Entretanto, o objetivo do WAS-TelEduc é diferente de todos os demais trabalhos encontrados na literatura. Nosso objetivo é acessar funcionalidades do ambiente de e-learning com requisições como: "Qual é a próxima atividade da agenda?" ou "Avise quando sair a nota da disciplina de Engenharia de Software”. Em outras palavras, pretendemos que o agente WAS acesse funcionalidades do ambiente a partir das redes sociais, de modo que os usuários possam obter certas informações sobre os seus cursos sem precisar logar no TelEduc. Supomos que essa concepção do WAS-TelEduc poderá trazer agilidade e comodidade às consultas do usuário, contribuindo para aumentar o acesso ao ambiente TelEduc e, consequentemente, para maior participação dos alunos.

\section{Avaliação de Viabilidade do WAS-TelEduc}

Apesar de termos encontrado trabalhos na literatura que descrevem a interação entre usuários de ambientes de e-learning e Chatterbots, não identificamos nenhum que apresente uma avaliação de viabilidade desse tipo de interação. Alguns trabalhos exploram essa ideia, mas nenhum deles procura responder perguntas como: "os usuários de ambientes de e-learning querem interagir com um agente em linguagem natural?" ou "os usuários acham útil esse tipo de recurso?", o que nos parece um ponto de partida fundamental quando se deseja desenvolver aplicações centradas nos usuários.

Nesta seção, apresentamos a avaliação da viabilidade sobre o uso do agente Write and Solve (WAS) para acessar informações do ambiente de e-learning TelEduc, aqui denominado de WAS-TelEduc. Nosso objetivo é saber se os usuários gostariam de acessar o TelEduc por meio de um agente como o WAS.

Elaboramos um questionário que foi respondido por 40 usuários, dentre eles 13 estudantes (33\%), 17 professores (43\%) e 10 profissionais de outras áreas (26\%), como fonoaudiologia, tecnologia da informação, enfermagem, etc. Esses dados mostram a heterogeneidade do público que utiliza o TelEduc e indicam que seu uso não se restringe ao ambiente acadêmico. O Gráfico 1(a) mostra quais papéis os usuários assumem ou já assumiram no TelEduc - $63 \%$ dos usuários já utilizaram o TelEduc como aluno, $43 \%$ como professor/formador, $10 \%$ como coordenador de curso e $12 \%$ como administrador do ambiente.

Procuramos coletar dados sobre o uso das redes sociais e dos dispositivos móveis por parte dos usuários. O Gráfico 1(b) mostra quais redes sociais os usuários mais utilizam. O Facebook é, de longe, a rede social mais utilizada entre os participantes de nossa pesquisa. O Google Talk, apesar de não ser uma rede social, foi incluído no questionário por ser utilizado por muitos usuários do Gmail como forma de comunicação em tempo real e pela sua característica textual que o torna interessante para ser utilizado com o agente WAS. Os resultados mostram que $30 \%$ dos entrevistados são usuários do GTalk.

Identificamos também a frequência com que os participantes utilizam as redes sociais: $82 \%$ deles utilizam mais de três vezes por semana, sendo que $60 \%$ dos usuários 
utilizam dispositivos móveis como tablets e celulares para acessar as redes sociais mais de uma vez por semana.

Gráfico 1 - (a) Papéis assumidos pelos usuários TelEduc; (b) Redes sociais mais utilizadas pelos usuários; (c) Opinião dos usuários sobre a utilidade em acessar 0 ambiente de e-learning via redes sociais.

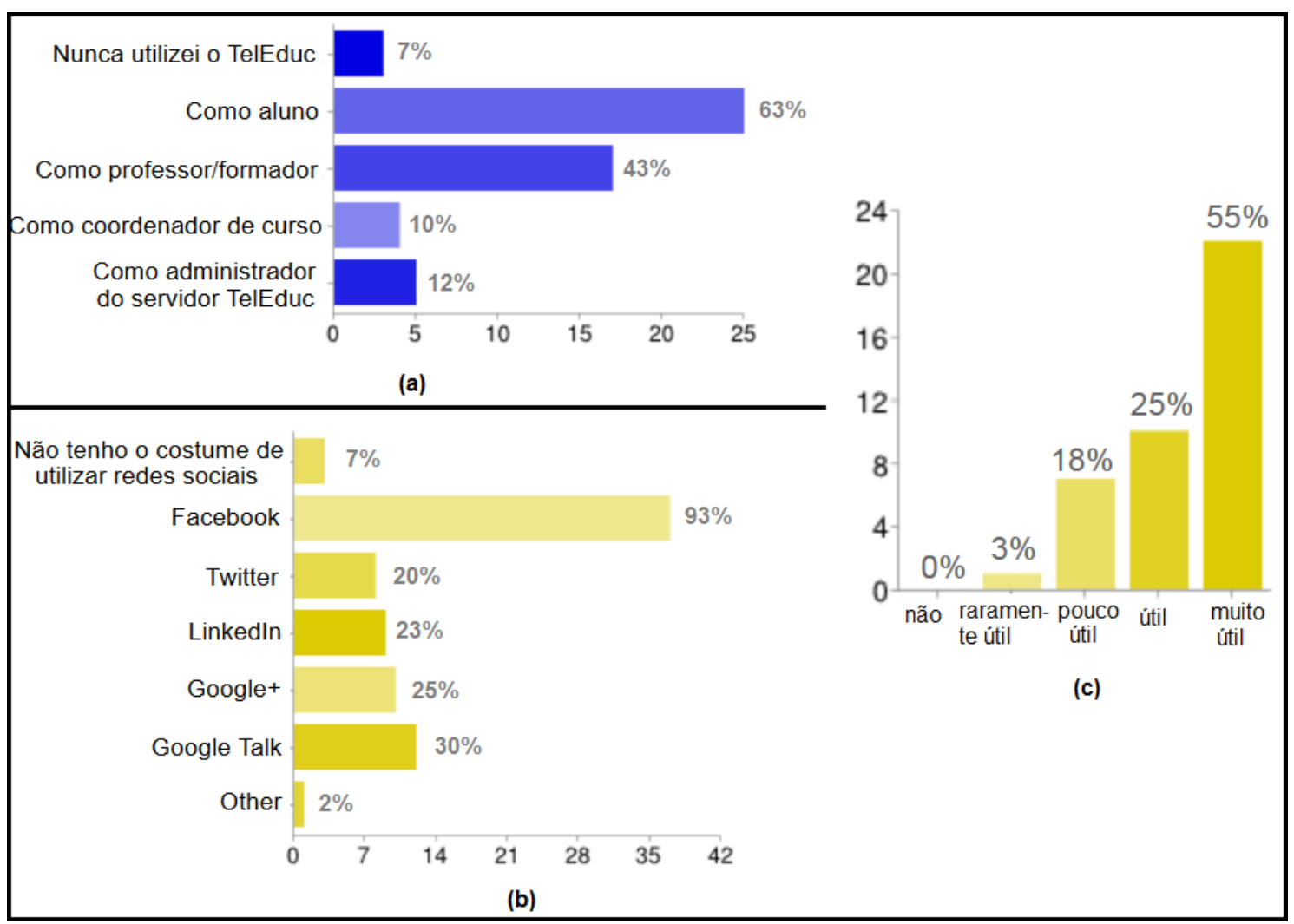

A respeito da assiduidade com que os usuários utilizam o ambiente TelEduc em períodos críticos, tais como, época de entrega de trabalhos, preparação de curso, etc; $63 \%$ dos usuários responderam que acessam o ambiente mais de três vezes por semana. Note-se que a frequência de acesso às redes sociais é maior, mesmo em períodos de intenso uso do ambiente de e-learning.

Com relação ao acesso ao ambiente TelEduc via dispositivos móveis, como tablets e celulares, apenas $15 \%$ dos usuários responderam que já utilizaram o ambiente nesses dispositivos, sendo que apenas $8 \%$ gostou da experiência. Acreditamos que a possibilidade de acessar o ambiente de e-learning por meio das redes sociais poderá aumentar o uso do TelEduc em dispositivos móveis, pois os dados mostram que os usuários estão acostumados a acessar as redes sociais com tais dispositivos, mas não têm esse mesmo hábito para acessar o ambiente TelEduc.

Procuramos também identificar se os usuários achariam útil acessar o ambiente TelEduc via redes sociais. O Gráfico 1(c) mostra que $80 \%$ deles acham útil ou muito útil. Perguntamos ainda se os usuários gostariam que o WAS acessasse seus cursos no TelEduc para fornecer informações enquanto estão logados nas redes sociais. Antes de responder a essa pergunta, convidamos os usuários para assistirem a um vídeo sobre o 
WAS, com o objetivo de compreenderem melhor seu objetivo, funcionamento e forma de interação ${ }^{12}$. A grande maioria dos usuários (88\%) respondeu que sim. Esse resultado responde a nossa pergunta inicial sobre o desejo dos usuários acessarem o ambiente de e-leaning usando um agente do tipo Chatterbot via redes sociais.

Investigamos ainda em quais redes sociais os usuários gostariam de ter o agente WAS como contato. O resultado foi similar ao Gráfico 1(b), isto é, os usuários gostariam de ter o WAS nas redes sociais que mais têm o costume de acessar Facebook 78\%, Gtalk 33\%, Google+23\%, LinkedIn 13\% e Twitter 8\%.

Para finalizar nossa pesquisa, pedimos a opinião dos usuários sobre a integração do TelEduc com o agente WAS. A seguir, transcrevemos algumas respostas que chamaram nossa atenção:

"Seria muito útil para o aluno poder contar com a tecnologia para seu aprendizado, já que (a rede social) é a ferramenta com a qual o aluno mais se identifica na atualidade." (opinião de um professor do ensino médio/técnico)

"Abro minha rede social todos os dias, porém esqueço-me de verificar as mensagens do TelEduc com a mesma frequência. O WAS poderia ser uma ferramenta que me avisa na rede social quando tiver mensagens, postagens ou novidades na agenda do TelEduc." (opinião de uma estudante graduada)

"Essa relação potencializa a utilização do próprio LMS. Torna-o utilizável e muito presente no cotidiano, já que atualmente boa parte de nosso tempo é 'vivido' dentro das redes sociais. Vivamos lá, também, o TelEduc." (opinião de um professor do ensino médio/técnico)

"Interessante e inovador, pois hoje tudo gira nas redes sociais e os dispositivos vivem conectados a elas, então porque não ficar conectado ao TeleEduc. Espero que dê certo." (opinião de um estudante de graduação)

\section{Discussão}

Com a popularização de dispositivos móveis juntamente com a adoção de planos móveis de acesso à Internet através de redes como a $3 \mathrm{G}$, podemos dizer que a ampla utilização de formatos de troca de mensagens como o SMS ou mesmo a mensageria instantânea (IM) são uma tendência. A principal motivação para o desenvolvimento do WAS é poder acessar informações de outros sistemas a partir das redes sociais, por meio de mensagens de texto simples. Acreditamos que a solução adotada pelo WAS pode ter um impacto bastante positivo e motivador para um público que cada vez mais "vive" nas redes sociais e está cada vez mais conectado ao mundo por meio de dispositivos móveis.

Neste artigo, analisamos a viabilidade de utilizar o agente WAS para acessar informações de um ambiente de e-learning, mais especificamente, o ambiente TelEduc. Entretanto, a integração com um agente desse tipo pode ser realizada em outros ambientes de e-learning similares, o que torna a avaliação de viabilidade aqui apresentada adequada para ser generalizada para outros ambientes.

\footnotetext{
${ }^{12} \mathrm{O}$ vídeo está disponível em http://www.writeandsolve.com.br/wascorp/acao
} 
O principal objetivo da pesquisa realizada foi responder a duas questões: "os usuários de ambientes de e-learning querem interagir com um agente em linguagem natural?" e "os usuários acham útil esse tipo de recurso?". De acordo com os resultados, a resposta a essas duas perguntas é sim, o que nos motiva fortemente a trabalhar na integração do agente WAS com o ambiente de e-learning TelEduc.

Além de responder as nossas perguntas iniciais, a avaliação de viabilidade também trouxe resultados que confirmam que as nossas expectativas estão alinhadas com os interesses dos usuários com relação a agilidade e comodidade para acesso ao ambiente TelEduc. A pesquisa mostra que a frequência de acesso às redes sociais é maior quando comparada ao ambiente de e-learning, mesmo em períodos de intenso uso do ambiente. Com base em nossos resultados, acreditamos que facilitar e agilizar o acesso ao ambiente de e-learning via redes sociais poderá aumentar a frequência de acesso ao ambiente e, consequentemente, incrementar a participação dos alunos.

Por fim, outro dado interessante está relacionado ao uso dos dispositivos móveis. A possibilidade de acessar o ambiente de e-learning por meio das redes sociais poderá aumentar o uso do TelEduc via dispositivos móveis, pois os dados de nossa pesquisa mostram que os usuários estão acostumados a acessar as redes sociais com tais dispositivos, mas não têm esse mesmo costume em relação ao ambiente TelEduc. Outros trabalhos nesta direção estão em andamento no âmbito do Projeto TelEduc [Silva et. al. 2013a; 2013b].

\section{Conclusões e Trabalhos Futuros}

$\mathrm{O}$ uso das redes sociais em conjunto com os ambientes de e-learning constitui uma nova tendência de uso das TDICs na educação. A maior parte dos trabalhos encontrada na literatura procura enriquecer os ambientes de aprendizagem com recursos das redes sociais. Em nossas pesquisas, procuramos adotar o caminho inverso, isto é, usar as redes sociais para acessar os ambientes de e-learning. Supomos que esse tipo de solução pode ter um impacto bastante positivo e motivador para o público da sociedade da informação atual em que é raro encontrar quem não acesse ao menos uma rede social.

Neste artigo descrevemos a avaliação de viabilidade com relação à integração do agente WAS e o ambiente TelEduc. Por meio do WAS-TelEduc pretendemos acessar funcionalidades do ambiente de e-learning a partir das redes sociais; assim, os usuários poderão obter informações sobre seus cursos com maior comodidade e agilidade.

A avaliação de viabilidade mostrou que os usuários desejam e acham útil tal concepção do WAS-TelEduc. Além disso, a avaliação apresentou dados que orientarão a integração do WAS com o ambiente de e-learning TelEduc. Por exemplo, o Gráfico 1(b) mostra que vale a pena iniciarmos a integração do TelEduc com os comunicadores instantâneos do Facebook e do Gtalk, pois estes são os mais utilizados pelos usuários.

Como trabalhos futuros, procederemos com questões relacionadas à concepção do WAS-TelEduc, tais como: Quais requisições os usuários gostariam de solicitar ao WAS-TelEduc?; Como gostariam de ser notificados na ocorrência de novos eventos em seus cursos?; Qual vocabulário será utilizado na interação? dentre outras. Por fím, pretendemos avaliar o impacto que essa nova tendência relacionada ao acesso a 
ambientes de e-learning via redes sociais pode ter na educação por meio de estudos de caso reais.

\section{Referências}

Batista, A. M.; Marietto, M. G. B.; Barbosa, G. O. Franca, R. S.; Kobayashi, G (2009). Multi-agent systems to build a computational middleware: A chatterbot case study. In: International Conference for Internet Technology and Secured Transactions (ICITST 2009), pp. 1-2.

Bickmore, T.; Giorgino, T (2006). Health dialog systems for patients and consumers. Journal of Biomedical Informatics, vol. 39, no. 5, pp. 556-571.

Cassell, J (2001). Embodied conversational agents: Representation and intelligence in user interfaces. AI Magazine, vol. 22, no. 4, pp. 67-84.

Cerceau, A. D. (1998) Formação a distancia de recursos humanos para informática educativa. Campinas, SP. Instituto de Computação, UNICAMP. (Dissertação de Mestrado).

Colazzo, L.; Molinari, A.; Villa, N. (2007) Towards a More Fluid Learning Environment Based on Virtual Communities. 10th IEEE International Conference on Advanced Learning Technologies, Sousse, Tunisia, 2010, pp. 742-743.

Figl, K.; Kabicher, S.; Toifl, K. (2008) Promoting social networks among computer science students. 38th Ann. Frontiers in Education Conf. (FIE 2008), Saratoga Springs, New York, 22-25 October 2008, pp. S1C-15-S1C-20.

Ghali, F.; Cristea, A. I. (2009) Authoring for E-Learning 2.0: a Case Study. In: 9th IEEE International Conference on Advanced Learning Technologies (ICALT 2009), Riga, Letônia, pp. 170-174.

Halimi, K.; Seridi, K.; Faron-Zucher, C (2011). solearn: a social learning network. IEEE International Conference on Computational Aspects of Social Networks (CASoN), 2011, Salamanca, Spain, pp. 130-135.

Kaeomanee, Y.; Dominic, D. D.; Rias, R. P. B. M (2012). Social Software Enhanced Elearning System to Support Knowledge Sharing among Students. IEEE International Conference on Computer \& Information Science (ICCIS), 2012, Kuala Lumpur, Malaysia, pp. 25-30.

Li, M.; Liu, Z. (2009) The role of online social networks in students e-learning experiences. Int. Conf. on Computational Intelligence and Software Engineering (CiSE 2009), Wuhan, China, 11-13 December 2009, pp. 1-4.

Mahapatra, R. P.; Sharma, N.; Trivedi, A.; Aman, C (2012). Adding interactive interface to E-Government systems using AIML based chatterbots. In: Proceedings of Sixth International Conference on Software Engineering (CONSEG 2012), pp.1-6, Sept. 2012.

Mikic, F.; Burguillo, J.; Llamas, M.; Rodriguez, D.; Rodriguez, E (2009). Charlie: An AIML-based chatterbot which works as an interface among INES and humans. In: 39th IEEE Frontiers in Education Conference, pp. 1-6. 
Moreno E. C.; Meza, M.; Arteaga J. M.; Padilla, A.; Padilla, A.; Rodriguez, F. A (2012). Use of chatterbot for accessing learning objects on mobile devices with a data mining search engine. In: 22nd International Conference on Electrical Communications and Computers (CONIELECOMP), pp. 134-137.

Oliveira, E. A.; Tedesco, P.; Chiu, T. P. T. F (2012). Creating personalized and distributed virtual learning spaces through the use of i-collaboration 3.0. In: Proceedings of the 12th international conference on Intelligent Virtual Agents (IVA'12). Berlin, Heidelberg: Springer-Verlag, pp. 477-479.

Rodrigues, J.J.P.C.; Sabino, F.M.R.; Zhou, L. (2001) Enhancing e-learning experience with online social networks. IET Communications, 2011, Vol. 5, Iss. 8, pp. 11471154. doi: 10.1049/iet-com.2010.0409.

Silva, A. C. da; Freire, F. M.; Rocha, H. V (2013a). Identifying Cross-Platform and Cross-Modality Interaction Problems in e-Learning Environments. In: International Conference on Advances in Computer-Human Interactions (ACHI), Nice, França. Proceedings of the Sixth International Conference on Advances in Computer-Human Interactions (ACHI), 2013. p. 243-249.

Silva, A. C. da ; Freire, F. M.; Arruda, A. V. P. ; ROCHA, H. V (2013b). Interaction Problems Accessing e-Learning Environments in Multi-touch Mobile Devices: a case study in TelEduc. In: MCCSIS - IADIS International Conference on e-Learning, Praga, República Theca. Proceedings of the IADIS International Conference on eLearning, 2013, p. 1999-206.

Sing, G. O.; Wong, K. W.; Fung, C. C.; Depickere, A (2006). Towards a more natural and intelligent interface with embodied conversation agent. In: Proceedings of the 2006 international conference on Game research and development (CyberGames'06). Australia: Murdoch University, pp. 177-183.

Takegata, S; Tanaka-Ishii, K (2010). Youbot: A simple framework for building virtual networking agents. In: Proceedings of the SIGDIAL 2010 Conference. Tokyo, Japan: Association for Computational Linguistics, pp. 273-276.

Wallace, R. S (2009). The anatomy of A.L.I.C.E. In: Parsing the Turing Test. R. Epstein, G. Roberts, and G. Beber (Eds). Springer Netherlands, pp. 181-210.

Weerawarna, N. T.; Haththella, H. M. H. R. B.; Ambadeniya, A.; Chandrasiri, L. H. S. S.; Bandara, M. S. L.; Thelijjagoda, S (2011). Cybermate artificial intelligent business help desk assistant with instance messaging services. In: 6th IEEE International Conference on Industrial and Information Systems (ICIIS), pp. 420424.

Weizenbaum, J (1966). Eliza - a computer program for the study of natural language communication between man and machine. Communications of the ACM, vol. 9, no. 1 , pp. 36-45. 